\title{
Have we misjudged the role of children in spreading COVID-19?
}

\author{
n Cite as: CMAJ 2020 September 21;192:E1102-3. doi: 10.1503/cmaj.1095897
}

Posted on cmajnews.com on September 3, 2020

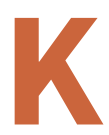
ids may play a bigger role in the spread of severe acute respiratory syndrome coronavirus-2 (SARSCoV-2) than initially suspected, according to Ontario's science advisory table on coronavirus disease 2019 (COVID-19).

"Early suggestions that children are considerably less important drivers of SARSCoV-2 transmission than adults are not confirmed by more recent research," the expert group cautioned in a science brief. "Children could play a relevant role in SARS-CoV-2 transmission and school closures may have been important contributors to the containment of the COVID-19 pandemic."

According to the latest evidence, kids older than 10 are just as likely to be infected or infect others with SARS-CoV-2. The picture is less clear when it comes to younger kids because of blind spots in the research, says Dr. Peter Juni, executive director of Ontario's COVID-19 science advisory table.

Children have been "dramatically underrepresented" in COVID-19 case counts because they are less likely than adults to show symptoms and therefore may escape detection, Juni explains. Especially early in the pandemic, "there were a lot of biased studies, and people were really uncritical of that."

For example, contact tracing studies that tested only kids with symptoms of COVID-19 were "obviously biased," given that young children are more likely to be asymptomatic, Juni says.

Seroprevalence studies can be equally misleading. "Schools were closed when the studies were done," Juni notes, so findings that children were less likely than adults to have antibodies against the virus may reflect reduced exposure at that time.
Kids may have some biological protection against SARS-CoV-2. The virus uses angiotensin-converting enzyme 2 (ACE2) receptors in the nasal mucosa as a doorway into the cells, and studies have found that young children have a lower density of those receptors.

Yet, Juni says, "nobody knows" if kids' poor adherence to physical distancing and other precautions cancel out any biological advantage. "Take my six-yearold - even the son of an epidemiologist, will he actually be able to do everything he needs to do to keep safe in school?"

A retrospective cohort study of an outbreak at an overnight youth camp found that younger children were at least as likely as older attendees to become infected with SARS-CoV-2. Meanwhile, young kids with COVID-19 have similar viral loads to older kids and adults, although it is unclear if they are equally infectious.

Misinterpretations of early research on school closures further confused the picture of kids' role in the pandemic, Juni says. An influential rapid review published in April found a lack of evidence to support closing schools. However, some media mistook that finding as proof that school closures don't work. "They mixed up a lack of evidence with a lack of effectiveness," Juni explains.

Other studies have since linked school closures with the containment of the virus, but it is impossible to separate the impact of those closures from other public health measures.

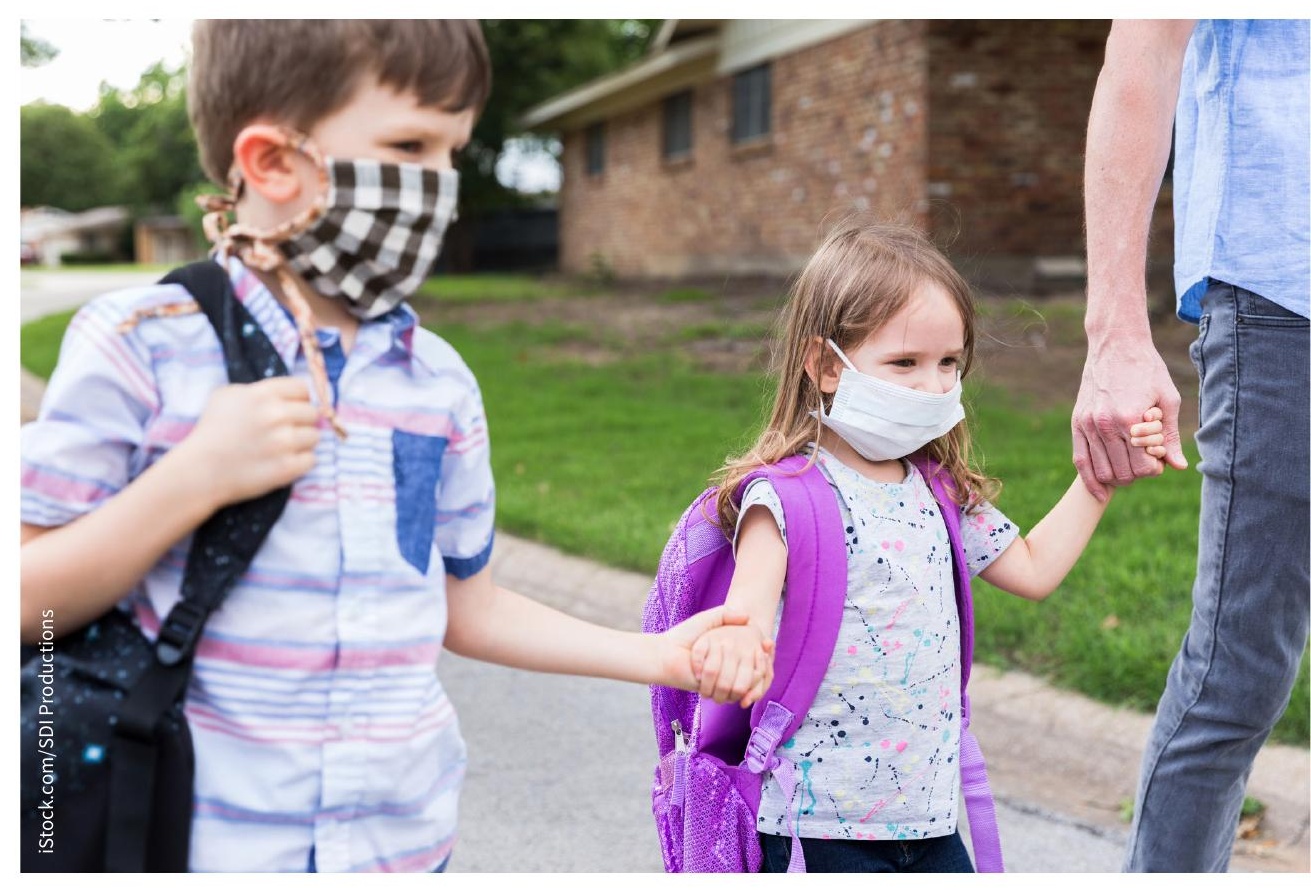

Experts caution that we may be underestimating kids' role in the COVID-19 pandemic. 
According to pediatricians Dr. Benjamin Lee and Dr. William Raszka, resolving the question of the role kids play in SARSCoV-2 transmission is "central to making informed public health decisions," including how to safely reopen schools.

In a recent article in Pediatrics, Lee and Raszka noted that transmission of the virus from children to adults outside of household settings "seems uncommon." They cited two examples of school outbreaks involving limited or no spread beyond the close contacts of initial cases. Such case studies "provide reassurance that school-based transmission could be a manageable problem."

According to Ontario's COVID-19 science advisory table, school closures may have played an important role in containing SARS-CoV-2. However, some European countries like Denmark have demonstrated that reopening schools can be safe, "provided that local transmission is low and appropriate measures are in place," such as reduced class sizes and well-ventilated classrooms.

Reopening without such measures "could be associated with a higher number of school outbreaks," as seen in Israel, the group warns.

Teachers and public health experts in Ontario and across Canada have raised alarm over large class sizes and poor ventilation under school reopening plans.

"The important thing is not to panic but take it seriously," Juni says. "There will be outbreaks in schools, so the more we keep class bubbles relatively small and isolated from each other, the better we will be able to shut down individual classes instead of having to close entire schools."

Lauren Vogel, CMAJ 